216

\section{IMPAIRED IN VIVO MITOCHONDRIAL KREBS CYCLE ACTIVITY FOLLOWING MYOCARDIAL INFARCTION ASSESSED USING HYPERPOLARIZED MAGNETIC RESONANCE SPECTROSCOPY}

M Dodd, ${ }^{1}$ H Atherton, ${ }^{1}$ C Carr, ${ }^{1}$ J Griffin, ${ }^{2}$ L Heather, ${ }^{1}$ D Stuckey, ${ }^{1}$ G Radda, ${ }^{1}$ $\mathrm{K}$ Clarke, ${ }^{1} \mathrm{D}$ Tyler ${ }^{1}{ }^{1}$ University of Oxford; ${ }^{2}$ University of Cambridge

\section{doi:10.1136/heartjnl-2013-304019.216}

An increasing body of evidence links alterations in cardiac metabolism with the progression of heart disease. Using the recently developed technique of hyperpolarized ${ }^{13} \mathrm{C}$ magnetic resonance spectroscopy, in vivo alterations in mitochondrial metabolism were assessed following myocardial infarction (MI). Hyperpolarization of $13 \mathrm{C}$ containing compounds can increase their signal by $>10,000$ fold over conventional methods.

MI with reperfusion surgery was performed on eleven female Wistar rats. Four sham animals were also prepared. Animals were given two hyperpolarized scans, of either $\left[1-{ }^{13} \mathrm{C}\right]$ or $\left[2-{ }^{13} \mathrm{C}\right]$ pyruvate, at 1,6 and 22 weeks post-MI. $\left[1-{ }^{13} \mathrm{C}\right]$ or $\left[2-{ }^{13} \mathrm{C}\right]$ pyruvate were hyperpolarized and dissolved in a GE prototype polarizer. $1 \mathrm{ml}$ of $80 \mathrm{mM}$ hyperpolarized pyruvate was injected over $10 \mathrm{~s}$ via a tail vein catheter into an anaesthetised rat positioned in a 7T MR scanner. Spectra were acquired every second for a $1 \mathrm{~min}$ following

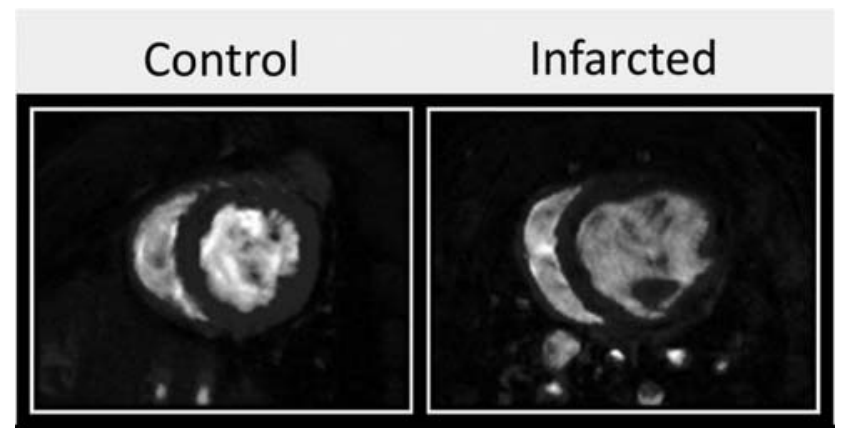

Figure 1 injection, using a $5^{\circ} \mathrm{RF}$ excitation pulse. Signal was localised to the heart using a custom ${ }^{13} \mathrm{C}$ RF surface coil. Metabolic alterations were correlated with ejection fraction (EF) assessed by echocardiography, at each timepoint to yield information on the interplay between cardiac function and mitochondrial metabolism.

One week post-MI, there were no detectable alterations in in vivo cardiac mitochondrial metabolism over the range of EFs observed. This is an early adaptive phase post-MI, where scar formation and remodelling of the heart are occurring. Six weeks post-MI, a novel finding in this study was impaired in vivo mitochondrial Krebs cycle activity, in addition to decreased flux into acetylcarnitine, which correlated with the EF. These changes were seen in the absence of any alterations in pyruvate dehydrogenase $(\mathrm{PDH})$ flux. Thus, in vivo alterations in Krebs cycle flux may indicate an early maladaptive phase in the metabolic derangement following MI.

By 22 weeks post-MI, alterations were also seen in PDH flux, which positively correlated with $\mathrm{EF}$, highlighting a reduction in glucose oxidation and Krebs cycle activity in the infarcted heart. At 22 weeks, biochemical analysis was performed on excised hearts, to further characterize the metabolic alterations accompanying MI. Enzyme activities of $\mathrm{PDH}$, citrate synthase, isocitrate dehydrogenase and carnitine acetyltransferanse positively correlated with EF Metabolomic analysis revealed reduced levels of Kerbs cycle intermediates.

The correlation between function and metabolism raises an interesting paradox; is the reduction in $\mathrm{PDH}$ and Krebs cycle activity due to a reduction in contraction and therefore a reduced energy requirement, or does the altered $\mathrm{PDH}$ and Krebs cycle activity lead to reduced energy levels meaning cardiac contraction is impaired? This study highlights the importance of assessing metabolism at multiple timepoints in vivo, and demonstrates the potential of hyperpolarized MRS for investigating the metabolic effects of progressive diseases, potentially in a clinical setting. 